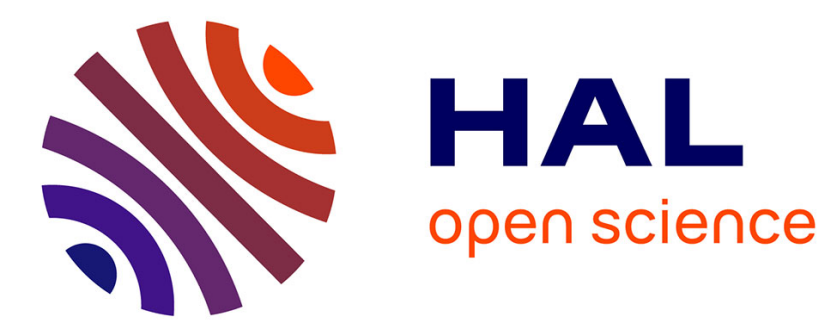

\title{
Retro-engineering state machines into PDDL domains
}

Maxence Grand, Humbert Fiorino, Damien Pellier

\section{To cite this version:}

Maxence Grand, Humbert Fiorino, Damien Pellier. Retro-engineering state machines into PDDL domains. IEEE International Conference on Tools with Artificial Intelligence, 2020, Baltimore (virtual conference), United States. pp.1186-1193, 10.1109/ICTAI50040.2020.00180 hal-03243892

\section{HAL Id: hal-03243892 \\ https://hal.science/hal-03243892}

Submitted on 31 May 2021

HAL is a multi-disciplinary open access archive for the deposit and dissemination of scientific research documents, whether they are published or not. The documents may come from teaching and research institutions in France or abroad, or from public or private research centers.
L'archive ouverte pluridisciplinaire HAL, est destinée au dépôt et à la diffusion de documents scientifiques de niveau recherche, publiés ou non, émanant des établissements d'enseignement et de recherche français ou étrangers, des laboratoires publics ou privés. 


\section{Retro-engineering state machines into PDDL domains}

\author{
$1^{\text {st }}$ Maxence Grand \\ Univ. Grenoble Alpes, LIG \\ 38000 Grenoble, France \\ Maxence.Grand@univ-grenoble-alpes.fr
}

\author{
$2^{\text {nd }}$ Humbert Fiorino \\ Univ. Grenoble Alpes, LIG \\ 38000 Grenoble, France \\ Humbert.Fiorino@imag.fr
}

\author{
$3^{\text {rd }}$ Damien Pellier \\ Univ. Grenoble Alpes, LIG \\ 38000 Grenoble, France \\ Damien.Pellier@imag.fr
}

\begin{abstract}
This paper presents a new algorithm based on grammar induction, called AMLSI (Action Model Learning with State machine Interactions), that retro-engineers planning domains described with Planning Problem Description Language (PDDL) by querying a state machine with action sequences and by observing the state transitions. AMLSI takes as input a training set of feasible and infeasible action sequences built from partial observations and returns a PDDL domain. A key issue for domain learning is the ability to plan with the learned domains. It often happens that a small learning error leads to a domain that is unusable for planning. Unlike other algorithms, we show that AMLSI is able to lift this lock by learning domains from partial observations with sufficient accuracy to allow planners to solve new problems.
\end{abstract}

\section{INTRODUCTION}

Many real world systems implicitly rely on state machines. In communicating systems, for example, each party has to follow the same communication protocol otherwise the system could deadlock. Each party follows a state machine where an action like sending or receiving a message puts the overall system into a new state. For instance, an ATM dispenses cash only when the machine is in a state where a card has been inserted and the PIN is verified.

Planning Domain Definition Language (PDDL) allows to model state machines and to plan action sequences achieving targeted goals. It is generally accepted that hand-encoding PDDL is difficult, tedious and error-prone. Moreover, the experts of the system to model are not always PDDL experts and vice versa. In order to tackle this issue, learning algorithms have been proposed to automatically generate PDDL domains. Many algorithms are based on large training datasets composed of successful plan traces and observed transition states. But, in some situations, these training datasets are hard or impossible to collect: for some industrial processes, physical simulators are available but only operate in real time, which can amount to hours/days for each simulated action. In other cases, available production planning datasets are obsolete due to product changes over time, or the production expertise is implicit human knowledge. Another important issue for these learning algorithms is the accuracy of learned domains. Accuracy evaluates the performances of the algorithms to learn International Planning Competition (IPC) domain benchmarks and to reuse them for planning. Accuracy is way more challenging than syntactical distance between benchmarks and learned domains because the accuracy oftenly depends on a few number of decisive predicates of the planning domain.

In this paper, we propose a learning algorithm, AMLSI (Action Model Learning with State machine Interactions), allowing to "retro-engineer" real world state machines as accurate PDDL domains: unlike many state-of-the-art approaches, AMLSI does not require a training dataset of plan traces to work. AMLSI proceeds by trial and error: it queries the system to retro-engineer with randomly generated action sequences, and it observes the state transitions of the system. For instance, in the ATM example, sequences of actions like inserting a card, typing a number, aborting money withdrawal etc. are tested. These action sequences can possibly be infeasible. No prior knowledge on correct sequences is required, and AMLSI observations can be partial.

A key idea in AMLSI is that retro-engineering real world state machines is analogous to learning regular grammars: we argue that (1) it is possible to learn a regular grammar by querying a real world state machine, and (2) to induce a PDDL domain from this regular grammar. The contributions of AMLSI are to generate (1) accurate PDDL domains from relatively (2) small datasets with (3) partial observations.

The rest of the paper is organized as follows. In section II we present the related works. Section III is the problem statement. In section IV we present some backgrounds on regular grammar induction. In section V, we detail the AMLSI algorithm. Finally, section VI evaluates the performance and the accuracy of AMLSI on IPC benchmarks.

\section{RELATED WORKS}

These works can be classified according to the input data of the learning process and the complexity of the language used in the output domain. The input data can be plan traces obtained by resolving a set of planning problems, partial planning domains to complete or random walks. The input data can be complete (states and actions), partial, completely blind,or noisy. As output, the learned planning domains can have different levels of expressivity: negative preconditions, static relations, numerical functions or conditional effects.

Many works takes as input a set of plan traces and a partial domain, and tries to incrementally refine this domain to complete it, as for instance, EXPO [1] and more recently RIM [2]. In practice, RIM constructs sets of "soft" and 


\begin{tabular}{|c|c|c|c|c|c|c|c|}
\hline & Input & Observations & Noise & Negative preconditions & Static relations & Numerical functions & Conditional effects \\
\hline EXPO & Partial domain, Plan traces & Complete & No & NA & NA & No & No \\
\hline RIM & Partial domain, Plan traces & Complete & No & NA & NA & No & No \\
\hline Observer & Plan traces & Complete & No & No & NA & No & No \\
\hline ARMS & Plan traces & Partial & No & NA & NA & No & No \\
\hline SLAF & Plan traces & Partial & No & NA & No & No & Yes \\
\hline Louga & Plan traces & Partial & No & Yes & Yes & No & No \\
\hline Plan-Milner & Plan traces & Partial & Yes & NA & No & Yes & No \\
\hline LOCM & Plan traces & No Observation & - & NA & No & No & No \\
\hline LOCM2 & Plan traces & No Observation & - & NA & No & No & No \\
\hline LOP & Plan traces & No Observation & - & $\overline{\mathrm{NA}}$ & Yes & No & No \\
\hline NLOCM & Plan traces & No Observation & - & NA & Yes & Yes & No \\
\hline IRALe & Random walk & Complete & Yes & NA & NA & No & No \\
\hline LSO-NIO & Random walk & Partial & Yes & No & Yes & No & No \\
\hline AMLSI & Random walk & Partial & No & Yes & Yes & No & No \\
\hline
\end{tabular}

TABLE I: State-of-the-art domain learning algorithms. For some algorithms, there is no indication on the ability of the algorithm to learn static relations and/or negative preconditions. These algorithms are rated NA for these criteria.

"hard" constraints between observed states and actions, which are solved with weighted MAX-SAT solvers to obtain the refined domain. In all these approaches, it is assumed that the observations are complete and noiseless.

A second group of works takes as input only plan traces. Most of them deal with partial observations (except Observer [3]). Among these approaches are ARMS [4], SLAF [5], Louga [6] or Plan-Milner algorithm [7]. ARMS gathers knowledge on the statistical distribution of frequent sets of actions in plan traces. It then forms a weighted propositional satisfiability problem (weighted SAT) and solves it with a weighted MAX-SAT solver. Unlike ARMS, SLAF is able to learn domains with conditional effects. To that end, SLAF relies on the building of logical constraint formula based on a direct acyclic graph representation. Then, Louga also takes as input plan traces, and work with partial and noiseless observations. However, Louga is able to learn domains with static properties and negative preconditions. Louga uses a genetic algorithm to learn action effects and an ad-hoc algorithm to learn action preconditions. Plan-Milner uses a classification algorithm based on inductive rule learning techniques: it learns domains with discrete numerical values from partial and noisy observations. Finally, the LOCM family of domain learning approaches [8]-[11] works without information about initial, intermediate and final states. These algorithms extract, from plan traces, parameterized automata representing the behaviour of each object of the domain. Then preconditions and effects are generated from these automata.

The last group of works takes as input a set of action sequences randomly generated. Random walk approaches like IRALe [12] deal with complete but noisy observations. IRALe is based on an online active algorithm to explore and to learn incrementally the domain with noisy observations. Others approaches such as LSO-NIO [13] are able to deal with both partial and noisy observations. LSO-NIO uses a classifier based on a kernel trick method to learn domains. It consists of two steps: (1) it learns a state transition function as a set of classifiers, and (2) it derives the domain from the parameters of the classifiers.

AMLSI differs from the state-of-the-art algorithms in several ways. Firstly, AMLSI works with both feasible and infea- sible action sequences while most approaches use only feasible action sequences or plan traces. To our best of knowledge, only IRALe and LSO-NIO use failures in action sequences, but AMLSI differentiates feasible and infeasible action sequences. In addition, AMLSI uses random walks to generate its training datasets of both feasible and infeasible action sequences, whereas most of the algorithms like ARMS, Louga and PlanMilner either only use plan traces or random walks generating feasible action sequences. Thirdly, in terms of expressivity, AMLSI learns PDDL domains including static relations in preconditions as well as negative preconditions. To the best of our knowledge, only Louga has the same expressivity but it cannot work with noisy observations. More importantly, AMLSI is the only algorithm able to learn planning domains accurate enough to allow planners to solve new planning problems (i.e. that are not in the training sets) with different level of partial observability (see Section VI).

\section{Problem Statement}

We work in the context of classical planning. World states $s$ are modeled as sets of propositions, and actions change the world states. For instance, in the gripper domain,

$$
\begin{aligned}
s= & \left(\text { at }- \text { robby } r_{1}\right) \wedge \neg\left(\text { at }- \text { robby } r_{2}\right) \wedge\left(\text { at } b r_{1}\right) \wedge \\
& \neg\left(\text { at } b r_{2}\right) \wedge(\text { free left }) \wedge(\text { free right }) \wedge \\
& \neg(\text { carry } b \text { right }) \wedge \neg(\text { carry b left })
\end{aligned}
$$

states that robby is in the room $r 1$ as well as the ball $b$, and robby's arms are both free.

Formally, let $S$ be the set of all the propositions modeling properties of world, and $A$ the set of all the possible actions in this world. A state $s$ is a subset of $S$ and each action $a \in A$ is a triple of proposition sets $\left(\rho_{a}, \epsilon_{a}^{+}, \epsilon_{a}^{-}\right)$, where $\rho_{a}, \epsilon_{a}^{+}, \epsilon_{a}^{-} \subseteq$ $S$, and $\epsilon_{a}^{+} \cap \epsilon_{a}^{-}=\emptyset . \rho_{a}$ are the preconditions of action $a$, that is, the propositions that must be in the state before the execution of action $a . \epsilon_{a}^{+}$and $\epsilon_{a}^{-}$are respectively the positive (add list) and the negative (del list) effects of action $a$, that is, the propositions that must be added or deleted in $s$ after the execution of the action $a$. In practise, actions are abstracted as operators in first-order logic Planning Domain Description Language. For instance move(? from ?to) stands for all the 


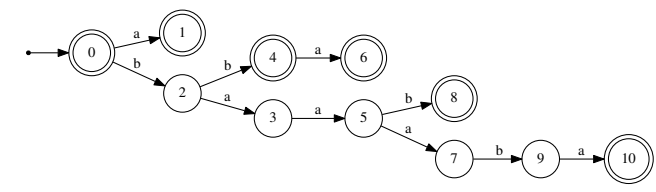

(a) The Prefix Tree Acceptor

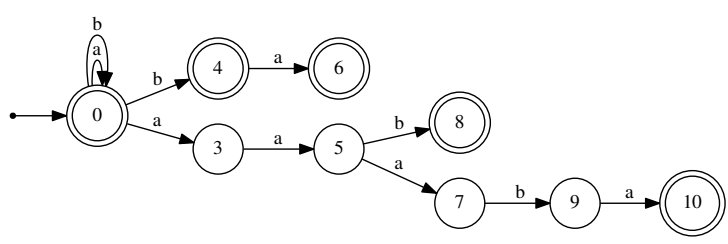

(c) Fusion of nodes 0 and 2

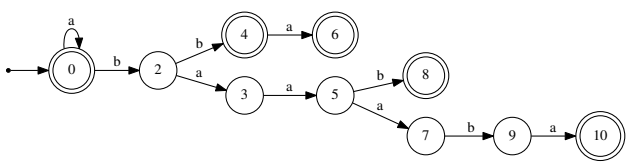

(b) Fusion of nodes 0 and 1
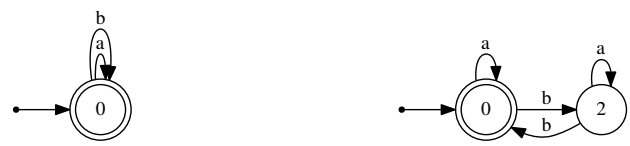

(d) Deterministic merge

(e) Final automaton

Fig. 1: An example of RPNI execution

possible groundings of the parameters ?from and ?to, and the actions move $\left(r_{1} r_{2}\right)$, move $\left(r_{2} r_{1}\right)$ etc.

Let $\gamma: S \times A \rightarrow S$ be the state transition function such that $s^{\prime}=\gamma(s, a)=\left(s \cup \epsilon_{a}^{+}\right) \backslash \epsilon_{a}^{-} \cdot \gamma(s, a)$ is defined if and only if $\rho_{a} \subseteq s$. Let $\pi=\left[a_{0}, a_{1}, \ldots, a_{n}\right]$ be a sequence of actions, and + the concatenation of two sequences. $\Gamma\left(s_{0}, \pi\right)$ is defined recursively as follows:

$$
\Gamma\left(s_{0}, \pi\right)= \begin{cases}{\left[s_{0}\right]} & \text { if } \pi=\emptyset \\ {\left[s_{0}\right]} & \text { if } \rho_{a_{0}} \nsubseteq s_{0} \\ {\left[s_{0}\right]+\Gamma\left(\gamma\left(s_{0}, a_{0}\right),\left[a_{1}, \ldots, a_{n}\right]\right)} & \text { otherwise }\end{cases}
$$

In this work, we assume that:

- the set of all the possible actions $A$ is known (for instance $A=\left\{\right.$ move $\left(r_{1} r_{2}\right)$, move $\left(r_{2} r_{1}\right), \operatorname{pick}\left(b \quad r_{1}\right.$ grip $)$, pick(b $r_{2}$ grip $), \operatorname{drop}\left(b r_{1}\right.$ grip $), \operatorname{drop}\left(b r_{2}\right.$ grip $\left.\left.)\right\}\right)$ and for all $a \in A,\left(\rho_{a}, \epsilon_{a}^{+}, \epsilon_{a}^{-}\right)$are unknown and must be learned,

- $\gamma$ is the state machine to model, and $\Gamma$ represents the observed states: $\left(s_{0}, \pi\right)$ is a query. If $\left(s_{0}, \pi\right)$ is sent to the state machine, it sends back a sequence of observable states $\Gamma\left(s_{0}, \pi\right)$. This kind of state machines is, for instance, an ATM on which a sequence of actions (inserting a credit card, a PIN number etc.) is tested, any process computing streams of user commands, an industrial process simulator or interviewed people ("what if in this state, we perform such and such action?") to collect their expertise on a process etc.

- the observations $\Gamma\left(s_{0}, \pi\right)$ are possibly partial. A partial observation is a state where some propositions are missing. For instance, in the gripper domain,

$$
\begin{aligned}
& \left(\text { at }- \text { robby } r_{1}\right) \wedge \ldots \wedge\left(\text { at } b r_{1}\right) \wedge \\
& \neg\left(\text { at } b r_{2}\right) \wedge \ldots \wedge(\text { free right }) \wedge \\
& \neg(\text { carry } b \text { right }) \wedge \neg(\text { carry b left })
\end{aligned}
$$

$\pi=\left[a_{0}, a_{1}, \ldots, a_{n}\right]$ is a feasible sequence given $s_{0}$ if and only if $\Gamma\left(s_{0},\left[a_{0}, a_{1}, \ldots, a_{n}\right]\right)=\left[s_{0}, s_{1}, \ldots, s_{n}\right]$ : both sequences have the same length. Therefore, $\pi=\left[a_{0}, a_{1}, \ldots, a_{n}\right]$ is an infeasible sequence given $s_{0}$ if and only if $\Gamma\left(s_{0},\left[a_{0}, a_{1}, \ldots, a_{n}\right]\right)=\left[s_{0}, s_{1}, \ldots, s_{i}\right]$ and $i<n$. Or, equivalently, $\left[a_{0}, a_{1}, \ldots, a_{i-1}\right]$ is feasible and $\left[a_{0}, a_{1}, \ldots, a_{i-1}, a_{i}\right]$ is infeasible (given $s_{0}$ ).

In this paper we tackle the following problem: by repeatedly querying a state machine with different (possibly infeasible) $\left(s_{0}, \pi\right)$ and observing $\Gamma\left(s_{0}, \pi\right)$ (possibly partial), is it possible to learn the state transition function $\gamma$ and express it as a PDDL domain?

\section{BACKGROUND ON REGULAR GRAMMAR INDUCTION}

A regular grammar is a grammar that can be represented as a deterministic finite automaton. Formally, a deterministic finite automaton is defined by $\Sigma=\left\langle A, N, n_{0}, \gamma\right\rangle$ where:

- $A$ is the input alphabet

- $N$ is the set of nodes

- $n_{0} \in N$ is the initial node

- $\gamma$ is the node transition function

A regular grammar is a possible representation of a planning problem, where the alphabet represents actions and nodes represents state labels. This representation is relevant to learn PDDL domains because regular grammar induction is a welldefined problem [14], many algorithms were proposed to solve it.

Among them, RPNI (Regular Positive and Negative Inferences) [15] is able to learn a deterministic automaton using positive and negative samples of words made of $A$ elements. RPNI is relevant because: (1) it is able to identify the class of the regular languages in the limit [14] in polynomial time, and (2) it is optimal, i.e. RPNI learns the smallest automaton accepting the positive sample and rejecting the negative sample, when the samples are characteristic [16].

RPNI takes as input $I_{+}$and $I_{-} . I_{+}$(resp. $I_{-}$) is the positive (resp. negative) sample, it is a set of positive (resp. negative) examples. RPNI returns an automaton $\Sigma$ accepting all the positive examples, and rejecting all the negative examples. 
Figure 1 shows an execution of the RPNI algorithm. RPNI starts by building the Prefix Tree Acceptor $P T A\left(I_{+}\right)$. $P T A\left(I_{+}\right)$is a deterministic finite automaton that accepts only all the positive examples. For instance, Figure 1a gives the PTA for $I_{+}=\{\lambda, a, b b, b b a, b a a b, b a a a b a\}$ and $I-=$ $\{b, a b, a b a\}$ ( $\lambda$ is the empty word). Then RPNI tries to merge nodes and retains only the automata compatible with $I_{-}$. In Figure 1b, RPNI merges nodes 0 and 1 . The resulting automaton is compatible with $I_{-}$, so RPNI retains it. Then RPNI tries to merge nodes 0 and 2 (see Figure $1 \mathrm{c}$ ). The new automaton is non-deterministic, so RPNI merges the nodes responsible for this non-determinism (see Figure 1d). This automaton is not compatible with $I_{-}$so it is not retained. Finally RPNI tries all the other nodes merge, and the final automaton is in Figure 1e.

\section{THE AMLSI ALGORITHM}

The AMLSI algorithm takes as input the set of action $A$ and the set of propositions $S$, builds two datasets by interactions with a state machine and returns a PDDL domain. The two datasets are: $I_{+}$(positive sample) the sample containing the feasible action sequences and the corresponding observations, and $I_{-}$(negative sample) the sample containing the infeasible action sequences. At a given state $s$, we query the system about the feasibility of an action $a$ randomly chosen in $A$. If $a$ is feasible, the current state is observed and we add $a$ to the current $\pi$. This random walk is iterated until $\pi$ reaches an arbitrary length, and added to $I_{+}$. If $a$ is infeasible in the current state, the concatenation of $\pi$ and $a$ is added to $I_{-}$. After building the datasets, the AMLSI algorithm is composed of three steps:

1) Grammar induction: AMLSI uses a variant of RPNI called RPNI-PC learning regular grammars representing $\gamma$ state transition functions.

2) PDDL Domain Generation: AMLSI generates the PDDL actions from the mapping of the learned grammar with the observed states.

3) PDDL Domain Refinement: AMLSI refines the action preconditions and effects. This refinement steps is necessary to address partial observations.

\section{A. Grammar induction: the RPNI-PC algorithm}

We propose the RPNI-PC (Regular Positive and Negative Inference constrained by Pairewise Constraints) algorithm to deal with specific constraints of actions in planning: pairwise constraints.

Pairwise constraints are pairs of actions $\left(a_{i}, a_{j}\right)$ that cannot be consecutives: action $a_{i}$ is never followed by action $a_{j}$. These pairwise constraints allow to simulate negative examples, and to ensure that all the pairs of consecutive actions in the induced automaton can possibly occur in the PDDL domain. These constraints are based on the fact that for an action to be feasible a certain number of resources must be produced (add list) and others must be consumed (del list). For instance, in the gripper domain, action move $\left(r_{1} r_{2}\right)$ will never be followed by action pick $\left(b r_{1}\right.$ grip) because $\left(a t-r o b b y r_{1}\right)$ must

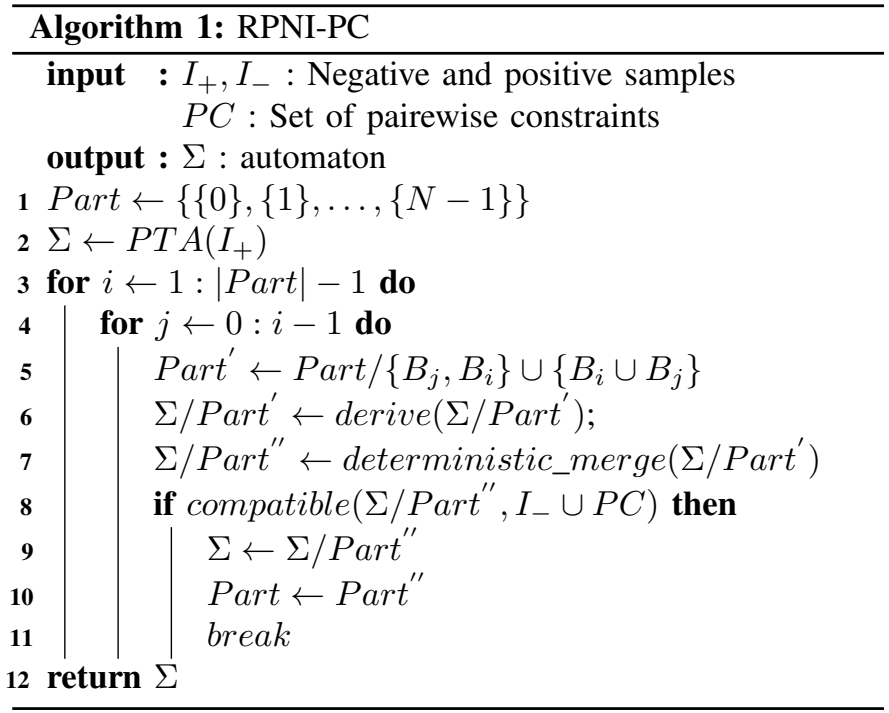

be true in order to execute action $p i c k\left(b r_{1}\right.$ grip), and, after the execution of action move $\left(r_{1} r_{2}\right)$, (at - robby $\left.r_{1}\right)$ is always false. Therefore it is impossible that action $\operatorname{pick}\left(b r_{1}\right.$ grip $)$ follows action move $\left(r_{1} r_{2}\right)$.

$P C$ is the set of pairewise constraints. To compute $P C$, we use the positive sample $I_{+}$. We assume that only the pairs present in the positive examples are possible pairs. Thus all the pairs that are not in $I_{+}$belong to $P C$ :

$$
\forall\left(a_{i}, a_{j}\right) \in A^{2},\left(a_{i}, a_{j}\right) \in P C \text { iff } \nexists \pi \in I_{+} \text {s.t. } \pi=\pi_{1}+\left[a_{i}, a_{j}\right]+\pi_{2}
$$

RPNI-PC (see Algorithm 1) starts by building the initial partition Part (line 1), and builds the PTA $\left(I_{+}\right)$(line 2). The partition is a set of subset of nodes. A subset of nodes is called Bloc, denoted B. If two nodes $n_{i}$ and $n_{j}$ are in the same bloc $B_{i}$, then the derivation of $\Sigma$ by Part denoted $\Sigma /$ Part is the automaton where these nodes are merged. Then RPNI-PC tries all nodes fusion (line 3-11). After merging two nodes $n_{i}$ and $n_{j}$ in the current partition (line 5), nodes are merged in the automaton with the function derive (line 6). If the automaton is non deterministic, the deterministic merge function merges the nodes responsible for the non-determinism (line 7). Then, RPNI-PC retains the merge if and only if the deterministic automaton rejects all the negative examples in $I_{-}$and all the pairewise constraints in $P C$ (line 8-11).

\section{B. PDDL Domain Generation}

The generation of the PDDL domain relies on tree steps described below.

- Step 1. Mapping of the observations into the automaton: Once the automaton is induced with RPNI-PC, we need to know which node of the automaton corresponds to which observed state. To that, we input in the automaton all the positive examples in $I_{+}$and map the pairs "node, action" in the automaton with the pairs "state, action" in $I_{+}$. There are two different mappings: the mapping (A)nte $\mu_{A}$ and the mapping (P)ost $\mu_{P}$. $\mu_{A}(n, a)$ (resp. $\mu_{P}(n, a)$ ) gives the state before (resp. 


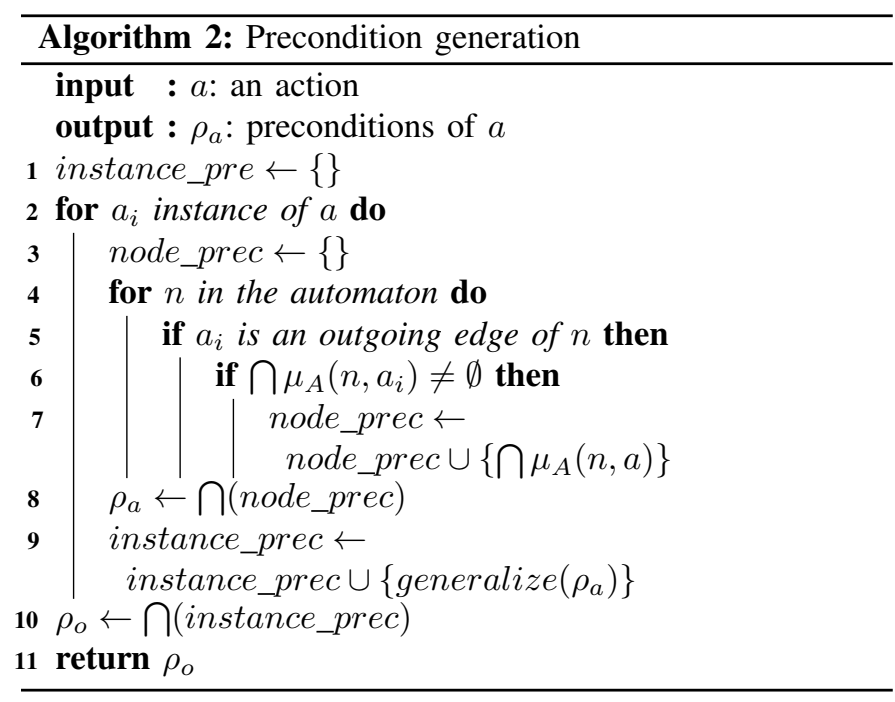

after) the execution of the transition $a$ in node $n$. Then, we compute a reduced mapping $\bigcap \mu_{A}\left(\operatorname{resp} \bigcap \mu_{P}\right)$, which contains the common propositions of all the states for a given $(n, a)$. For instance, in gripper domain: $\left(a t b r_{1}\right)$ is in $\bigcap \mu_{A}\left(0, \operatorname{pick}\left(b r_{1}\right.\right.$ grip $\left.)\right)$ iff $\left(a t b r_{1}\right)$ is in all $\mu_{A}\left(0, p i c k\left(b r_{1}\right.\right.$ grip $\left.)\right)$. Likewise, we remove from $\bigcap \mu_{A}\left(0, p i c k\left(b r_{1}\right.\right.$ grip $\left.)\right)$ and $\bigcap \mu_{P}\left(0, p i c k\left(b r_{1}\right.\right.$ grip $\left.)\right)$ all the propositions whose parameters are not a subset of (b $r_{1}$ grip), the parameters of pick $\left(b r_{1}\right.$ grip) (classical planning assumption [17]).

- Step 2. Generation of the action preconditions: The algorithm 2 describes how AMLSI generates action preconditions. To learn the preconditions of an action, we compute the reduced mapping of all the nodes where an action $a$ is feasible. At line $8, \rho_{a}$ is the reduced mapping of all the $\mu_{A}$ mappings of the action $a$. The generalization of $\rho_{a}$ at line 9 replaces all the constant symbols by variables (for instance, ( $b r_{1}$ grip) by (?obj ?room ?gripper)).

- Step 3. Generation of the action effects: Effect generation is analogous to precondition generation with the difference that we use $\mu_{A}(n, a)$ and $\mu_{P}(n, a)$ to compute a reduced mapping of $\epsilon_{a}^{+}$, the propositions that are never in $\mu_{A}(n, a)$ and always in $\mu_{P}(n, a)$, and $\epsilon_{a}^{-}$, the propositions that are always in $\mu_{A}(n, a)$ and never in $\mu_{P}(n, a)$

\section{PDDL Domain Refinement}

The last step is to refine the PDDL domain. This step is composed of two sub-steps that are iterated until convergence of the refined domain.

- Step 1. Refinement of the action effects: This step ensures that the generated operators allow to regenerate the induced grammar. We use the reduced mappings $\bigcap \mu_{A}$ to verify that for each couple of consecutive actions $a_{i}$ and $a_{j}$, the effects of the action $a_{i}$ generate the preconditions of action $a_{j}$. If it is not the case, we add in the effects of $a_{i}$ the propositions satisfying the preconditions of $a_{j}$.
For instance, suppose we have $n^{\prime}=\gamma\left(n\right.$, move $\left.\left(r_{1} r_{2}\right)\right)$ and $n^{\prime \prime}=\gamma\left(n^{\prime}, \operatorname{pick}\left(b r_{2}\right.\right.$ grip $\left.)\right)$. Now suppose we have $\neg\left(\right.$ at - robby $\left.r_{2}\right) \in \bigcap \mu_{A}\left(n\right.$, move $\left.\left(r_{1} r_{2}\right)\right)$, (at robby $\left.r_{2}\right) \in \rho_{\text {pick }\left(b r_{2} \text { grip }\right)}$ and $\left(a t-\right.$ robby $\left.r_{2}\right) \notin$ $\epsilon_{\text {move }\left(r_{1} r_{2}\right)}^{+}$. We need to have $\left(a t-\right.$ robby $\left.r_{2}\right) \in$ $\epsilon_{\text {move }\left(r_{1} r_{2}\right)}^{+}$in order to make $\gamma\left(n, \operatorname{move}\left(r_{1} r_{2}\right)\right)$ and $\gamma\left(n^{\prime}, \operatorname{pick}\left(b \quad r_{2}\right.\right.$ grip $\left.)\right)$ feasible. Thus, we add (at robby ?to) to $\epsilon_{\text {move }}^{+}$?from ?to) .

- Step 2. Refinement of the action preconditions In this step, we assume like [4] that the propositions of the negative effects must be in the action preconditions. Thus, for each negative effect of the operator, we add the corresponding proposition in the preconditions. For instance, suppose $\neg($ at - robby ?from $) \in \epsilon_{\text {move }(\text { from ?to) }}^{-}$, then (at robby ?from $) \in \rho_{\text {move(?from ?to) }}$ after refinement.

Since effect refinement depends on the preconditions, and precondition refinement depends on the effects, we iterate these two steps until convergence. They converge because the adding of preconditions is limited by the effects, and the adding of effects is limited by the preconditions of the next action in the induced automaton (In our experiments, see Section VI, less than 10 iterations are needed to converge).

\section{EXPERIMENTS}

Our experiments ${ }^{1}$ are based on 5 IPC domains: Blocksworld, Gripper, Hanoi, Sokoban and Neg-Elevator. Neg-Elevator is a modified version of Elevator with negative preconditions to show AMLSI ability to learn them. All the used benchmarks are STRIPS domains. Table II shows our experimental setup.

We deliberately chose the size of the test sets larger than the learning sets to show AMLSI ability to learn accurate domains with small datasets. The training and test sets are generated as explained in Section V.

In the training sets, we generate positive action sequences with a length randomly chosen between 10 and 20 . In the test sets, we generate positive action sequences with a length randomly chosen between 1 and 100. $I_{-}$is bigger than $I_{+}$ because it is more likely to generate infeasible actions.

We test each IPC domain with 3 different initial states over five runs, and we used five seeds randomly generated for each run. For each IPC domain, we generate partial observations by randomly removing a fraction of the propositions of the states. All tests were performed on an Ubuntu 14.04 server with a multi-core Intel Xeon CPU E5-2630 clocked at 2.30 $\mathrm{GHz}$ with $16 \mathrm{~GB}$ of memory.

\section{A. Evaluation Metrics}

We evaluated AMLSI with three different metrics. The first metric, the syntactical error, is the most used metric in the literature. The two other metrics, the FScore and the accuracy are more specific to our approach. The FScore allows to know if the learned domain is able to generate the state machine.

\footnotetext{
${ }^{1}$ Our experimental setup can be found in https://www.dropbox.com/sh/ y9p48mp7siacqzq/AADAzVmUV8nq9P8WOBXPVhJMa?dl=0
} 


\begin{tabular}{|c|c|c|c|c|c|c|c|c|c|c|c|c|c|c|c|c|c|c|c|c|c|c|}
\hline Domain & \#Operators & \#Predicates & \#Objects & \#Actions & \#Propositions & $\left|I_{+}\right|$ & $\left|I_{-}\right|$ & $\left|\pi_{+}\right|$ & $\left|\pi_{-}\right|$ & $\left|E_{+}\right|$ & $\left|E_{-}\right|$ & $\left|e_{+}\right|$ & $\left|e_{-}\right|$ \\
\hline Blocksworld & 4 & 5 & 3 & 18 & 16 & 30 & 2421.3 & 15 & 8.3 & 100 & 26209.5 & 49 & 33.6 \\
\hline Gripper & 3 & 4 & 5 & 10 & 8 & 30 & 1168.1 & 15.2 & 8.3 & 100 & 12940.3 & 50.7 & 33.7 \\
\hline Hanoi & 4 & 7 & 6 & 24 & 25 & 30 & 3011.3 & 14.8 & 8.2 & 100 & 34780.7 & 50.6 & 33.8 \\
\hline Sokoban & 2 & 4 & 14 & 26 & 42 & 30 & 3637 & 15 & 8.2 & 100 & 40464.7 & 50.4 & 33.8 \\
\hline Neg Elevator & 4 & 6 & 5 & 8 & 13 & 30 & 1050.7 & 15.1 & 8.6 & 100 & 13086.6 & 51 & 35.7 \\
\hline
\end{tabular}

TABLE II: Benchmark domain characteristics (from left to right): number of operators, number of predicates, number of objects in each initial states, number of actions in each initial states, number of propositions in each initial states, average size of $\left|I_{+}\right|$and $\left|I_{-}\right|$training sets, the average length of the positive (resp. negative) training sequences $\pi_{+} \in I_{+}$(resp. $\pi_{-} \in I_{-}$), average size of $\left|E_{+}\right|$and $\left|E_{-}\right|$test sets, the average length of the positive (resp. negative) test sequences $e_{+} \in E_{+}$(resp. $\left.e_{-} \in E_{-}\right)$.

\begin{tabular}{|c|c|c|c|c|c|}
\hline Domain & Algorithm & FScore(\%) & \#Nodes & \#Transitions & Compression level \\
\hline \multirow{2}{*}{ Blocksworld } & RPNI & 43.9 & 22 & 48.4 & 21.8 \\
& RPNI-PC & 53 & 23 & 43.4 & 19.7 \\
\hline \multirow{2}{*}{ Gripper } & RPNI & 100 & 23 & 16 & 58.9 \\
& RPNI-PC & 100 & 23 & 16 & 58.9 \\
\hline \multirow{2}{*}{ Hanoi } & RPNI & 21.6 & 25.2 & 61.6 & 16.7 \\
& RPNI-PC & 39.4 & 27.8 & 51.5 & 16.7 \\
\hline \multirow{2}{*}{ Sokoban } & RPNI & 61.7 & 20.1 & 50.7 & 29.4 \\
& RPNI-PC & 70.1 & 20.6 & 40 & 29.1 \\
\hline \multirow{2}{*}{ Neg Elevatpr } & RPNI & 21.8 & 24.2 & 45.1 & 19.1 \\
& RPNI-PC & 24.6 & 24.5 & 44.4 & 18.9 \\
\hline
\end{tabular}

TABLE III: Induced automaton characteristics (from left to right): algorithm used to induce automaton, FScore, average number of nodes, average number of transitions, compression level, i.e. average number of observed states per nodes.

The accuracy is the most important metric. It shows if the learned domain is able to solve new planning problems.

- Syntactical Error : The syntactical error error $(a)$ for an action $a$ is defined as the number of extra or missing predicates in the preconditions $\rho_{a}$, the positive effects $\epsilon_{a}^{+}$and the negative effects $\epsilon_{a}^{-}$divided by the total number of possible predicates [18]. The syntactical error for a domain with a set of actions $A$ is: $E_{\sigma}=\frac{1}{|A|} \sum_{a \in A} \operatorname{error}(a)$.

- FScore: This metric is initially used for pattern recognition and binary classification [19]. Nevertheless, it can be used to evaluate the quality of a learned grammar. Indeed, a grammar is equivalent to a binary classification system labeled with $\{1,0\}$. For grammars we can assume that the sequences belonging to the grammar are data labeled with 1 , and non-grammar sequences are data labeled with 0 . This metric is therefore able to test to what extent the learned domain $D$ can regenerate the regular grammar. A domain $D$ can regenerate a regular grammar if $D$ accept, i.e. regenerate all positive test sequences $e \in E^{+}$and reject all test negative sequences $e \in E^{-}$. Formally, the FScore is computed as follows: F-Score $=\frac{2 . P . R}{P+R}$ where $R$ is the recall, i.e. the rate of sequences $e$ accepted by the ground truth domain that are succesfully accepted by the learned domain, computed as follows: $R=\frac{\left\{e_{E} \in E^{+} \mid \operatorname{accept}(D, e)\right\} \mid}{|E|}$ and $P$ is the precision, i.e. the rate of sequences $e$ accepted by the learned domain that are sequences accepted by the ground truth domain, computed as follow: $P=$ $\left|\left\{e \in E^{+} \mid \operatorname{accept}(D, e)\right\}\right|$ ${\left.\overline{\mid\left\{e \in E^{+}\right.} \mid \operatorname{accept}(D, e)\right\} \cup\left\{e \in E^{-} \mid \operatorname{accept}(D, e)\right\}}$.

- Accuracy: This metric quantifies to what extent learned domains are able to solve planning problems [2]. Most of the works addressing the problem of learning planning domains uses the syntactical error to quantify the performance of the learning algorithm. However, domains are learned to be used for planning, and it often happens that one missing precondition or effect, which amount to a small syntactical error, makes them unable to solve planning problems. Formally, the accuracy $A c c=\frac{N}{N^{*}}$ is the ratio between $N$, the number of correctly solved problems with the learned domain, and $N^{*}$, the total number of problems to solve. The accuracy is computed over 20 problems. Although rarely used, we argue that the accuracy is the most important metric because it measures to what extent a learned domain is useful in practise for planning. In the experiments, we solve the test problems with Fast Downward v19.06 [20]. Plan validation is realized with the automatic validation tool used in the IPC competition VAL [21].

\section{B. Results}

In this section we perform an ablation study. Also, we don't compare AMLSI's results with baseline. Indeed, comparisons with other approaches in the literature are difficult due to differences in the learning settings. Table III compares automata characteristics learned with RPNI and our variant RPNI-PC. Figure 2 compares the results obtained for 4 variants of AMLSI:

1) Base: Grammar induction is done with RPNI and no refinement step is carried out.

2) Base + refine: Grammar induction is done with RPNI and a refinement step is carried out.

3) Base + PC: Grammar induction is done with RPNI-PC and no refinement step is carried out.

4) Base $+P C+$ Refine: Grammar induction is done with RPNI-PC and a refinement step is carried out.

First of all, Table III shows that the RPNI-PC algorithm learns better automata than RPNI. This is due to the fact that RPNI generally learns automata with fewer nodes and more transitions than the automata learned with RPNI-PC. For instance, for Sokoban, RPNI learns automata with 20.1 nodes and 50.7 transitions while RPNI learns automata with 20.6 nodes and 40 transitions. As a consequence, the automata accept a larger number of negative examples in the test set $E_{-}$. 

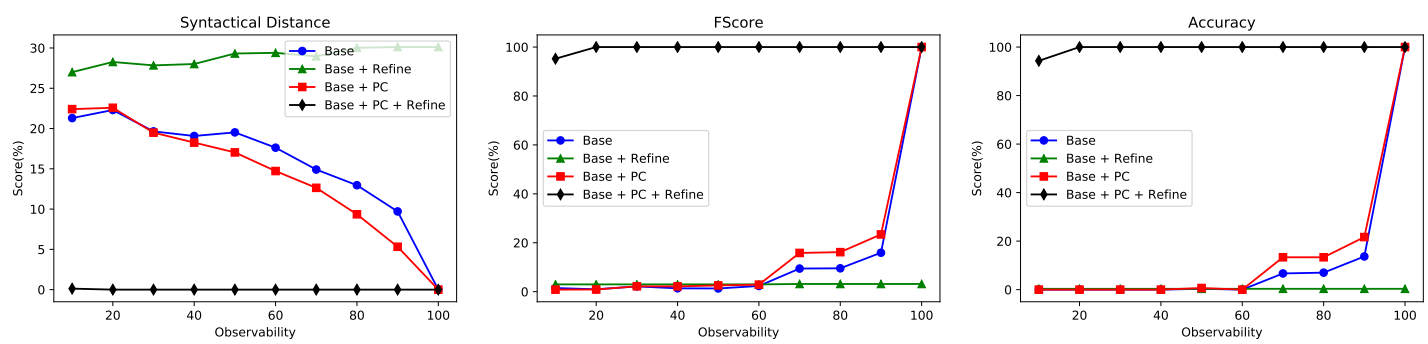

(a) Blocksworld
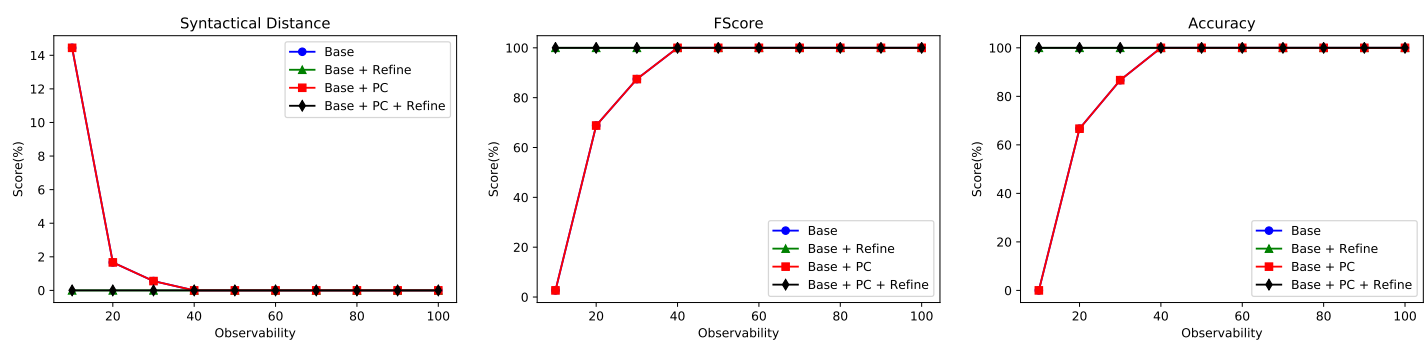

(b) Gripper
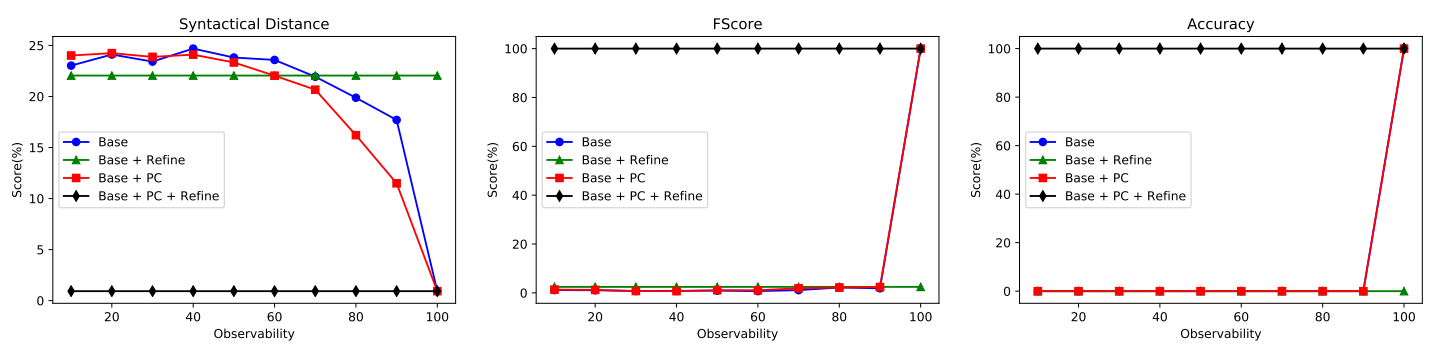

(c) Hanoi
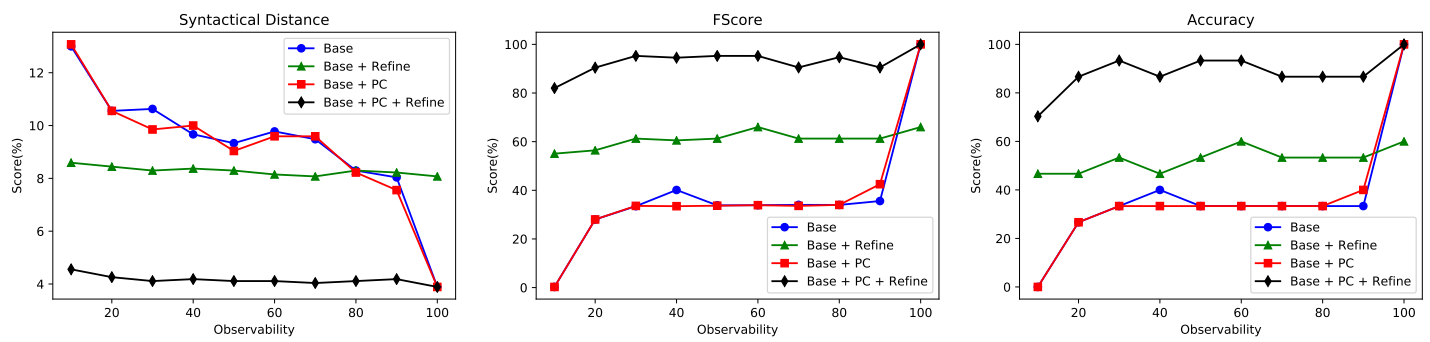

(d) Sokoban
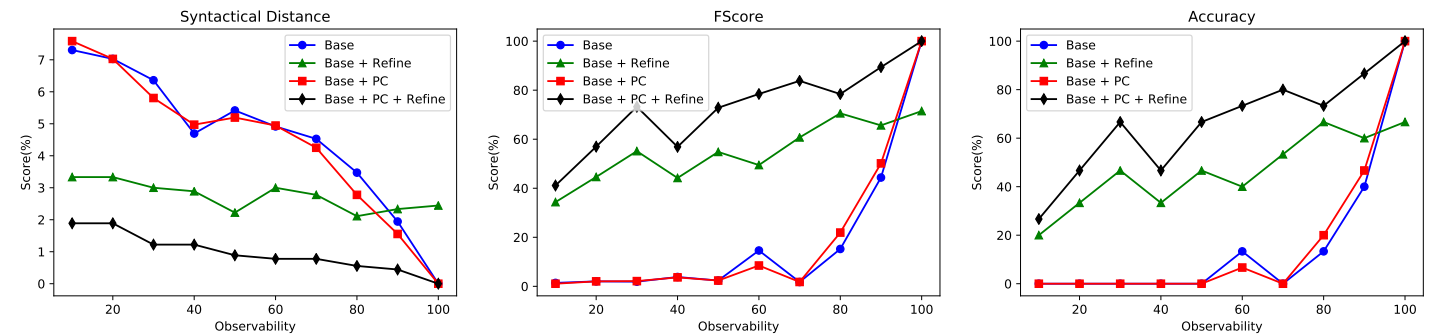

(e) Neg Elevator

Fig. 2: Learnt domains metrics with different levels of observability 
The figure 2 shows that the variant Base $+\mathrm{PC}+$ Refine gives the best results. For each domain, this variant gives the optimal domain (FScore $=100 \%$ and accuracy $=100 \%$ ) when the observations are complete. In addition, for the Gripper and Hanoi domains, BASE + PC + Refine always gives the optimal domain whatever the level of observability. Also, for the Blocksworld domain, BASE + PC + Refine gives the optimal domain when at least $20 \%$ of the propositions are present. In addition, we can observe that for the Sokoban and NegElevator domains, BASE + PC + Refine only learns the optimal domain when the observations are complete. Nevertheless, for these two domains, we have a high FScore (always greater than $80 \%$ for Sokoban, and greater than $60 \%$ when at least $50 \%$ of the propositions are present for NegElevator) and a high accuracy (greater than $80 \%$ when at least $20 \%$ of the propositions are present for Sokoban, and greater than $60 \%$ when at least $50 \%$ of the propositions are present for NegElevator). Note that for Hanoi and Sokoban, the syntactical error is not 0 while accuracy and FScore are optimal. This is because AMLSI learns preconditions that are not in the IPC domains. When we compare the variants Base and Base $+\mathrm{PC}$, we notice that Base + PC gives better results. It comes from the fact that automata learned with RPNI have fewer states than automata learned with RPNI-PC. As a consequence, we have a greater number of states per node, and therefore the mapping step removes too much information when the automata have been learned with RPNI.

Finally, we can note that Base + Refine is the only variant that can never learn the optimal domain. This is due to the fact that the automata learned with RPNI have a large number of false transitions, which induces extra effects and preconditions during the refinement step.

\section{CONCLUSION}

In this paper we have presented AMLSI, a novel algorithm to learn PDDL domains. Our objective is to retro-engineer state machines querying them and collecting partial observations. AMLSI is composed of three steps. In the first step, AMLSI induces a regular grammar. The second step is the generation of the PDDL operators, and the last step refines the generated operators. Our experimental results show that AMLSI is both performant and accurate enough to use learned domains for planning even with incomplete observations.

In future works, AMLSI will be extended to learn PDDL domain with noisy observations and more expressive planning languages such as Hierarchical Task Networks.

\section{ACKNOWLEDGMENT}

This research is supported by the French National Research Agency under the "Investissements d'avenir" program (ANR15-IDEX-02) through the Cross Disciplinary Program CIRCULAR.

\section{REFERENCES}

[1] Y. Gil, "Learning by experimentation: Incremental refinement of incomplete planning domains," in Machine Learning, Proceedings of the Eleventh International Conference, 1994, pp. 87-95.
[2] H. H. Zhuo, T. A. Nguyen, and S. Kambhampati, "Refining incomplete planning domain models through plan traces," in Proceedings of the 23rd International Joint Conference on Artificial Intelligence, IJCAI, 2013, pp. 2451-2458.

[3] X. Wang, "Learning by observation and practice: An incremental approach for planning operator acquisition," in Machine Learning, Proceedings of the Twelfth International Conference on Machine Learning, 1995, pp. 549-557.

[4] Q. Yang, K. Wu, and Y. Jiang, "Learning action models from plan examples using weighted MAX-SAT," Artif. Intell., vol. 171, no. 2-3, pp. 107-143, 2007.

[5] D. Shahaf and E. Amir, "Learning partially observable action schemas," in The Twenty-First National Conference on Artificial Intelligence and the Eighteenth Innovative Applications of Artificial Intelligence Conference, 2006, pp. 913-919.

[6] J. Kucera and R. Barták, "LOUGA: learning planning operators using genetic algorithms," in Knowledge Management and Acquisition for Intelligent Systems - 15th Pacific Rim Knowledge Acquisition Workshop, PKAW, 2018, pp. 124-138.

[7] J. Á. Segura-Muros, R. Pérez, and J. Fernández-Olivares, "Learning numerical action models from noisy and partially observable states by means of inductive rule learning techniques," in KEPS, 2018, pp. 46-53.

[8] S. Cresswell, T. L. McCluskey, and M. M. West, "Acquisition of objectcentred domain models from planning examples," in Proceedings of the 19th International Conference on Automated Planning and Scheduling, ICAPS, 2009.

[9] S. Cresswell and P. Gregory, "Generalised domain model acquisition from action traces," in Proceedings of the 21st International Conference on Automated Planning and Scheduling, ICAPS, 2011.

[10] P. Gregory and S. Cresswell, "Domain model acquisition in the presence of static relations in the LOP system," in Proceedings of the TwentyFifth International Conference on Automated Planning and Scheduling, ICAPS, 2015, pp. 97-105.

[11] P. Gregory and A. Lindsay, "Domain model acquisition in domains with action costs," in Proceedings of the Twenty-Sixth International Conference on Automated Planning and Scheduling, ICAPS, 2016, pp. 149-157.

[12] C. Rodrigues, P. Gérard, and C. Rouveirol, "Incremental learning of relational action models in noisy environments," in Inductive Logic Programming - 20th International Conference, ILP, 2010, pp. 206-213.

[13] K. Mourão, L. S. Zettlemoyer, R. P. A. Petrick, and M. Steedman, "Learning STRIPS operators from noisy and incomplete observations," in Proceedings of the Twenty-Eighth Conference on Uncertainty in Artificial Intelligence, 2012, pp. 614-623.

[14] E. M. Gold, "Language identification in the limit," Information and Control, vol. 10, no. 5, pp. 447-474, 1967.

[15] J. Oncina and P. García, "Inferring regular languages in polynomial update time," in Pattern Recognition and Image Analysis: Selected Papers from the IVth Spanish Symposium. World Scientific, 1992, vol. 1, pp. 49-61.

[16] P. Dupont, "Incremental regular inference," in Grammatical Inference: Learning Syntax from Sentences, 3rd International, 1996, pp. 222-237.

[17] R. Fikes and N. J. Nilsson, "STRIPS: A new approach to the application of theorem proving to problem solving," Artif. Intell., vol. 2, no. 3/4, pp. 189-208, 1971.

[18] H. H. Zhuo, Q. Yang, D. H. Hu, and L. Li, "Learning complex action models with quantifiers and logical implications," Artif. Intell., vol. 174, no. 18, pp. 1540-1569, 2010.

[19] C. J. V. RIJSBERGEN, Information Retrieval, 2nd ed. ButterworthHeinemann, 1979

[20] M. Helmert, "The Fast Downward planning system," Artif. Intell., vol. 26, pp. 191-246, 2006.

[21] R. Howey and D. Long, "Val's progress: The automatic validation tool for pddl2. 1 used in the international planning competition," in Proceedings of ICAPS Workshop on the IPC 2003, 2003, pp. 28-37. 